\title{
On the Relation Between Seismic Moment and Stress Drop in the Presence of Stress and Strength Heterogeneity
}

\author{
Raul Madariaga \\ Institut de Physique du Globe, Université de Paris VI, 75230 Paris Cedex 05. France
}

\begin{abstract}
The seismic moment is related by definition to the average slip on the fault plane of an earthquake. Here we derive an exact expression for the seismic moment in terms of a general heterogeneous stress drop distribution and the geometry of the fault of a complex event. We find that the seismic moment is proportional to a weighted integral of the stress drop on the fault. The weight in this linear relationship is the slip for a hypothetical event with the same source geometry but uniform stress drop. This relationship between seismic moment and stress drop depends on geometry. In particular, for multiple sources the weight is reduced by factors of the order of $\rho / R$, where $\rho$ is the radius of a typical subfault and $R$ is the radius of the total source area. As a consequence of these results we find that for a given stress drop, a simple fault generates a larger seismic moment than a multiple fault of the same total surface. Conversely, for a given moment and source area, a complex event would need higher stress drops on the subfaults than a simple smooth fault. We test these results with three rectangular models of faulting. The first is a simple, smooth fault with uniform stress drop. The second model is a simple fault with zero stress drop in the central section of the fault. The last model is a complex event where the central section of the fault remains unbroken. We show that the last two models are difficult to distinguish from their far-field radiation.
\end{abstract}

\section{INTRODUCTION}

The seismic moment, first determined by $A k i$ [1966] for the Nigata earthquake of 1964 , has become one of the most important source parameters. Kanamori and Anderson [1975] and Kanamori [1977] have presented a list of seismic moments for about 50 large earthquakes, while Hanks [1977] has summarized more than 400 seismic moment determinations for small- to medium-size events. The seismic moment was introduced from the representation theorem of dislocations as equivalent double couple sources [Burridge and Knopoff, 1964]. The representation theorem yields a definition of the seismic moment in terms of the slip at the fault and the source area. It is also important to express the seismic moment in terms of dynamic source parameters like the stress drop in order to invert the latter from seismic observations. This has been done in the past using specific fault models with uniform stress drop and simple geometries [Knopoff, 1958; Keilis-Borok, 1959]. The use of these models has led to the remarkable result that the calculated stress drops vary very little and are limited to the range from 1 to 100 bars [Aki, 1972; Thatcher and Hanks, 1973; Kanamori and Anderson, 1975; Hanks, 1977].

Several recent observations indicate, however, that many events are in fact complex or multiple events (Wyss and Brune [1967]; Hanks [1974]; Wu and Kanamori [1975]; Fukao and Furumoto [1975]; J. A. Rial, unpublished manuscript, 1978; H. Kanamori and G. Stewart, unpublished manuscript, 1977; among others). Direct evidence of the segmentation of faults has been reported by Spottiswoode and McGarr [1975] for shocks in deep gold mines in South Africa. Complex events have also been proposed in the numerical modeling of faulting with variable strength by Das and $A k i$ [1977] and Mikumo and Miyatake [1978]. Models proposed to explain source complexity may be broadly divided into two classes. First, models where the complexity is attributted to heterogeneity of stress drop due either to the nonuniformity of tectonic stress, of frictional stress, or of both. A massive, localized stress drop in the initial source region was proposed by Hanks [1974] for the San Fernando earthquake; a similar model was proposed by Burdick and Mellman [1976] for the Borrego Mountain earth-

Copyright (C) 1979 by the American Geophysical Union.

Paper number 8 B0926.

0148-0227/79/008B-0926\$01.00 quake. Kanamori and Stewart [1978] have interpreted the complexity of the Guatemala earthquake of 1976 by a highly nonuniform stress drop along the fault. Following them, we shall call 'asperity model' a model where source complexity is due to heterogeneous stress drop. The other, alternative model which we shall call the 'barrier model' has been proposed by Das and $A k i$ [1977]; in this model, complexity is due to the presence of unbreakable barriers along the fault plane. In this case the fault is not a simple, connected fault but a set of subfaults distributed on the fault plane. This model emphasizes the important role of strength in controlling and eventually stopping the growth of rupture on the fault. Each of the preceding models puts emphasis on either one of the two fundamental parameters that control the fracture of heterogeneous material: strength and stress heterogeneity.

From the previous discussion of observations and models, it appears that it would be very interesting to study the relation between stress drop, source geometry, seismic moment, and seismic radiation in the presence of heterogeneity of stress and source complexity. Madariaga [1977] has shown that in two dimensions the stress drop determined by the usual assumptions is not a true average of the stress drop on the fault. Here we shall derive a general relationship between seismic moment and stress drop for complex faults and heterogeneous stress field. We shall also compare numerical solutions for an asperity and a barrier model of source complexity.

\section{The General Relation of $M_{0}$, Stress Drop, AND GEOMETRY}

The seismic moment is usually defined in the form

$$
M_{0}=\mu \int_{S} d S D(x, y)=\mu \overline{D S}
$$

where $\mu$ is the rigidity, $D$ the offset of the fault, $S$ its total area, and $\bar{D}$ the average slip. As defined in (1) the seismic moment is a scalar: it is the moment of one of the couples in the doublecouple representation of a point dislocation [Burridge and Knopoff, 1964]. Implicit in the definition is the assumption that the fault is plane. For curved fault surfaces, or for non- 
coplanar multiple sources, the seismic moment should be defined as a tensor whose components are [Kostrov, 1974]

$$
M_{i j}=\mu \int_{S}\left(D_{i}(\mathbf{r}) n_{j}+D_{j}(\mathbf{r}) n_{i}\right) d S
$$

where $\mathrm{r}$ is the position vector on the fault, $\hat{n}=\left(n_{x}, n_{y}, n_{z}\right)$ is the unit normal, and $D$ is the slip vector at the point $r$. In (2) it was assumed that the faults do not open (i.e., D. $\hat{n}=0$ ). The surface $S$ may be curved, or it may be a collection of smaller faults in the case of multiple earthquakes.

The basis of this work is Betti's reciprocity theorem for static elastic fields. Let us consider two general elastic static states of the same elastic, possibly nonuniform body of volume $V$ and surface $S$. The surface $S$ includes the external surface $S_{\infty}$, which will be taken at infinity, and a collection of internal surfaces $S^{l}$ which we shall eventually close to form cracks. A first elastic state of displacement $u_{i}$ and stresses $\sigma_{i j}$ and a second one of displacements $v_{i}$ and stresses $\tau_{t j}$ are considered. We shall assume that there are no body forces in $V$. Then the elastic reciprocity theorem may be written in the form

$$
\int_{S} \tau_{l j} u_{l} n_{j} d S=\int_{S} \sigma_{i j} v_{l} n_{j} d S
$$

where $n_{l}$ are the components of the external normal to $S$. Let us now close the internal surfaces $S^{i}$ to form cracks. We choose on each of these cracks a negative and a positive side. We define the normal to the negative side as the reference normal. Then the identity (equation (3)) may be written as

$$
\begin{aligned}
\int_{S_{\infty}} \tau_{i j} u_{i} n_{\jmath} d S+\int_{\Sigma} \tau_{l j} \Delta u_{i} n_{\jmath} d S & \\
& =\int_{S_{\infty}} \sigma_{i j} v_{i} n_{j} d S+\int_{\Sigma} \sigma_{i j} \Delta v_{l} n_{\jmath} d S
\end{aligned}
$$

where $\Sigma$ is the collection of all internal surfaces $S^{i}, \Delta v_{t}=v_{l}^{+}-$ $v_{i}^{-}$, and $\Delta u_{i}=u_{i}^{+}-u_{i}^{-}$are the slips at the cracks for each elastic field. We now let the surface $S_{\infty}$ tend to infinity. If we take zero stress as the reference stress level, the integrals on $S_{\infty}$ will generally be finite. Yet it is well known that the absolute stress level does not affect seismic radiation; only stress changes do. For this reason we take the initial stress $\sigma_{i j}{ }^{0}$, or $\tau_{l j}{ }^{0}$, before the earthquake as the reference stress. In this case, $\tau_{l j}$ and $\sigma_{i j}$ are the stress changes due to slip at the faults. They, and the displacements fields $u_{l}, v_{l}$, decrease like $R^{-2}$ at infinity, and the integrals on $S_{\infty}$ may be dropped from (4). Let us remark that, as noticed by Savage [1969], in the earth, $\sigma_{i j}{ }^{\circ}$ and $\tau_{l j}{ }^{0}$ are of internal origin so that the elastic reciprocity theorem (equation (3)) does not apply to them. A second remark is that $S_{\infty}$ may be taken as the surface of the earth; for shallow earthquakes this may require modifications of the fields $v_{t}, \tau_{t j}$ used below.

Let us derive now an expression for the seismic moment (2) in terms of the stress drops. For that purpose we take $u_{i}, \sigma_{i j}$ to be the actual elastic field due to an earthquake; then

$$
\Delta u_{i}=D_{i} \quad \sigma_{i j} n_{j}=\Delta \sigma_{l} \quad \text { on } S^{i}
$$

where $D$ is the slip and $\Delta \sigma$ the stress drop at the fault $S^{\boldsymbol{t}}$. For $v_{i}, \tau_{i}$, we take the following:

$$
\begin{gathered}
\Delta v_{i}=E_{l}^{l} \quad \text { on } \quad S^{i} \\
\tau_{i j} n_{\jmath}=\mu n_{l} \delta_{i k} \quad \text { on } \quad S^{i}
\end{gathered}
$$

where $E_{l}{ }^{l}$ will be the slip determined from the solution of the crack problem with stress drop $\mu n_{l} \delta_{i k}$ and $\delta_{i k}$ is Kröneker's symbol. Replacing (5) and (6) in the reciprocity theorem (4), we find

$$
\mu \int_{\Sigma} n_{l} D_{k} d S=\int_{\Sigma} \Delta \sigma_{l} E_{l}^{l} d S
$$

Repeating the process with another solution of (6), where $\tau_{i j} n_{j}$ $=\mu n_{k} \delta_{l l}$, and adding, we finally find

$$
M_{l k}=\int_{\Sigma} \Delta \sigma_{l}\left(E_{l}{ }^{\imath}+E_{l}^{k}\right) d S
$$

where $\Sigma$ is the collection of all fault segments $S^{i}$. The expression (7) defines the seismic moment tensor in terms of the stress drop $\Delta \sigma_{l}$ in a completely general case. But this definition uses weighting functions $E_{l}{ }^{l}$ which themselves have to be obtained from the solution of crack problems. This may seem not to be very useful. However, in most problems of interest in seismology the slips $E_{l}^{l}$ are already known from standard solutions in fracture mechanics. For example, for plane faults the stress drop in $(6 b)$ is constant, and solutions are known for many simple fault shapes and for some multiple cracks. We shall use these solutions to define the seismic moment in several seismologically interesting problems.

\section{Simple Plane Faults With Heterogeneous STress Drop}

Assuming that slip is parallel everywhere on a plane fault, the seismic moment tensor may be written in the simpler form

$$
M_{l k}=M_{0}\left(n_{l} \nu_{k}+n_{k} \nu_{l}\right)
$$

where $n_{l}$ and $\nu_{k}$ are the cosine directors of the fault hormal and the slip vector, respectively, and $M_{0}$ is given by (1). From (7) we find the alternative expression

$$
M_{0}=\int_{\Sigma} \Delta \sigma_{l} E_{l} d S
$$

where $E_{l}$ is the slip calculated for a crack of the same shape as the studied fault, but with a uniform stress drop $\Delta \tau_{\imath}=\mu \nu_{l}$. Equation (9) yields a general relationship between seismic moment and stress drop for plane faults. It is valid for faults of any geometry, including multiple faults and heterogeneous stress drop on the fault. But the weighting function $E_{t}$ has to be obtained from the solution of an auxiliary crack problem with uniform stress drop. Let us consider first simple faults, i.e., faults without 'barriers' or unbroken patches. In this case, $E_{t}$ is known for several simple source shapes. It may also be readily calculated by finite element methods. Here, because of its mathematical simplicity, we shall assume that the source area is an ellipse; this is a rough approximation to some aftershock areas. A solution for an elliptical fault with uniform stress drop may be derived from Eshelby [1957]. The slip is found as

$$
E_{l}(x, y)=A_{l} W\left(1-\frac{x^{2}}{L^{2}}-\frac{y^{2}}{W^{2}}\right)^{1 / 2}
$$

where we choose the axis $x$ in the semimajor axis direction; $L$ and $W$ are the semimajor and semiminor axes, respectively. The nondimensional constants $A_{t}$ are a function of the ellipticity $\epsilon=W / L$ and are shown in Figure 1 for longitudinal $A_{x}$ or transverse $A_{y}$ slip. The constants $A_{t}$ are smoothly varying functions of the ellipticity, which vary at most by a factor of about 2 . In the case of a circular fault $L=W=R,(10)$ reduces to the well-known result 


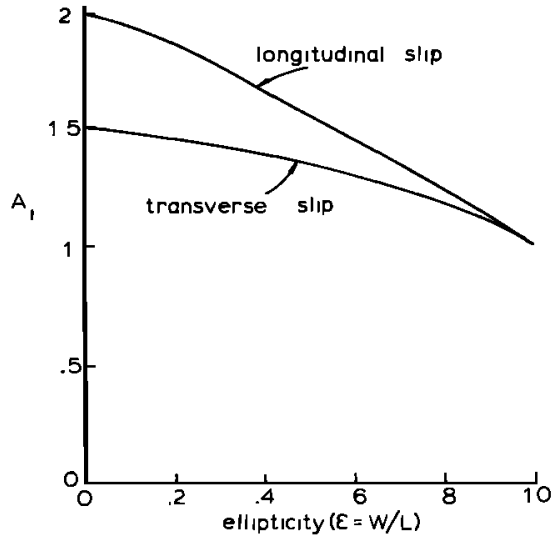

Fig. 1. Coefficient $A_{i}$ for elliptical faults (equation 10)) as a function of ellipticity $\epsilon=W / L$. Longitudinal slip is along the major axis of the fault, while traverse slip is across the minor axis.

$$
E_{i}(r)=\frac{24}{7 \pi} R\left(1-r^{2} / R^{2}\right)^{1 / 2}
$$

where $r$ is the radial variable [Eshelby, 1957; Keilis-Borok, 1959].

The expression (9) is valid for a general stress drop distribution on the fault and yields a precise relation between seismic moment and stress drop for elliptical faults. We may give it a more conventional form:

$$
M_{0}=C_{t}\left(\Delta \sigma_{t}\right) W S
$$

where now $C_{t}={ }_{3}^{3} A_{t}$ is a nondimensional constant which depends on the direction of slip at the fault and the ellipticity of the fault. It varies from 0.728 for circular faults to a maximum of 1.33 for longitudinal slip on a very long fault. The estimated stress drop

$$
\langle\Delta \sigma\rangle=\frac{3}{2 S} \int_{\Sigma} \Delta \sigma\left(1-\frac{x^{2}}{L^{2}}-\frac{y^{2}}{W^{2}}\right)^{1 / 2} d S
$$

is an average of the stress drop with a weighting function that emphasizes the stress drop near the center of the fault. The fault appears to be stiffer near the ends and softer toward the center of the fault. Equation (12) is the one most commonly used to invert stress drop from estimations of $M_{0}$ and $S$. A usual approximation is that the fault is circular, in which case $W=R$ and $C_{i}=0.728$. Therefore, it is $\langle\Delta \sigma\rangle$ that is estimated from observations. In the special case of smooth faulting with uniform stress drop $\Delta \sigma$, the estimated stress drop $\langle\Delta \sigma\rangle=\Delta \sigma$. For heterogeneous stress drops, $\langle\Delta \sigma\rangle$ will in general differ from the true average stress drop

$$
\overline{\Delta \sigma}=\frac{1}{S} \int_{\Sigma} \Delta \sigma d S
$$

The difference will be larger if the stress drop is larger near the edges of the fault. Extreme examples are the constant dislocation models (like Haskell's) where stress drop is infinite near the edges of the fault ( $R$. Madariga, manuscript in preparation, 1979); in that case, $\overline{\Delta \sigma}$ is unbounded, while $\langle\Delta \sigma\rangle$ is finite. In less extreme cases, the estimated stress drop $\langle\Delta \sigma\rangle$ will probably yield a good approximation to $\overline{\Delta \sigma}$.

The asperity model may be discussed with this theory. In this model, due to preseismic slip or foreshocks there are large stress concentrations in the vicinity of unbroken asperities of the fault. The earthquake releases these stress concentrations, and a smooth final stress distribution results. This model predicts highly heterogeneous stress drop so that the estimated stress drop $\langle\Delta \sigma\rangle$ will underestimate the stress drop at the asperities. Let us assume, as an example, that identical asperities cover a surface $S_{a}$ of the source area $S$. Assuming a stress drop $\Delta \sigma_{a}$ at the asperities and no stress drop in the rest of the fault plane, we find

$$
\langle\Delta \sigma\rangle \simeq \overline{\Delta \sigma}=\Delta \sigma_{a} S_{a} / S
$$

where, for purposes of illustration, we have neglected the difference between $\langle\Delta \sigma)$ and $\overline{\Delta \sigma}$. Thus the estimated stress drop underestimates the stress drop at the asperities by the ratio of the total asperity surface to the total source area.

\section{Complex, Multiple Plane Faults}

As mentioned in the introduction, several observations seem to indicate that earthquakes might in fact be complex suites of smaller events. Observational evidence of this type of source heterogeneity has been reviewed by $A k i$ et al. [1979]. They have interpreted these observations in terms of the barrier model of Das and Aki [1977]. In this model, segments of the fault remain unbroken after the main event. Intuitively, we see that these unbroken segments act as pins on the fault so that the slip will be highly heterogeneous. Simultaneously, large stress concentrations appear at the unbroken segments. These stress concentrations may be thought as stress rises or negative stress drops.

In order to discuss the seismic moment of these events we shall make several additional simplifying assumptions. First, we shall assume that all the multiple faults are coplanar. This assumption is also generally made in observational work. If this is not assumed, the moment tensor may not be written in terms of a single scalar $M_{0}$ as in (8). For multiple coplanar faults the seismic moment is again given by the expression (9). But now $E_{l}$ is the slip produced by a constant stress drop $\Delta \tau_{i}=$ $\mu \nu_{i}$ in each one of the subfaults of the complex event. This slip is necessarily smaller than the slip calculated for a single source of the same total area. Thus, for a given stress drop on the subfaults, the seismic moment will be larger for a single fault than for a multiple fault of the same equivalent area. When using (12) to estimate $\langle\Delta \sigma\rangle$ in the usual way, the actual stress drop in every subfault will be underestimated. To illustrate this problem, we shall assume that the actual event is made of $N$ smaller fractures of area $S^{t}$. We shall also assume that each of these subfaults is of circular shape. $E_{l}$ is the slip in a coplanar array of circular cracks with identical, uniform stress drop. The solution of this problem depends on the actual separation between faults and their spatial arrangement. For purposes of illustration we shall neglect the interaction between faults, assuming that they are independent. This will probably underestimate the slip on each fault by factors that depend on the distance between neighboring faults. The slip $E^{i}$ in each subfault is of the form (11) with $R=R_{i}$, the radius of one of the subfaults.

The total seismic moment for this complex event may be written as

$$
M_{0}=\frac{24}{7 \pi} \sum_{i} R_{\imath} \int_{S_{t}} \Delta \sigma\left(1-r^{2} / R_{i}\right)^{1 / 2} d S
$$

where the sum is over all the subfaults. We may rewrite this equation in the slightly different form

$$
M_{0}=\frac{16}{7 \pi} \sum_{i} R_{i}\langle\Delta \sigma\rangle_{l} S_{i}
$$

where $\langle\Delta \sigma\rangle_{i}$ is a weighted average of the form (13) of the stress drop in each of the subfaults. Compared to the simple crack 


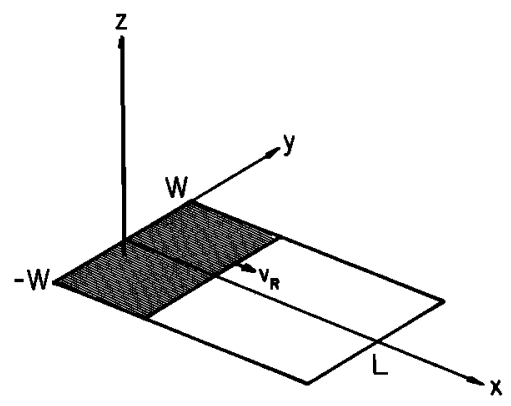

Fig. 2. Geometry of the rectangular fault model. Rupture starts at $x=0$ simultaneously across the width $W$ of the fault. Rupture velocity is constant $v_{R}=0.75 v_{S}$ and parallel to the longitudinal axis of the fault. In all the models the total fault length $L=2 W$.

model of stress drop $\langle\Delta \sigma\rangle=\langle\Delta \sigma\rangle_{t}$ and total surface $S=\sum S_{t}$, the seismic moment (17) is smaller by a factor of $R_{l} / R$ than that of the simple fault. We may see this more clearly if we assume that all the subfaults are identical, with radius $R_{t}=\rho$ and stress drop $\langle\Delta \sigma\rangle=\hat{\sigma}$ and that the total area $S=\sum_{l} S_{t}$. In this case the seismic moment (17) has the simpler expression

$$
M_{0}=\frac{16}{7 \pi} \rho \hat{\sigma} S
$$

This is to be compared to the seismic moment of a simple circular fault of area $S$ (and radius $R$ ) with apparent stress drop $(\Delta \sigma)$ :

$$
M_{0}=\frac{16}{7 \pi} R\langle\Delta \sigma\rangle S
$$

Assuming now that the stress drop on the simple circular fault is $\hat{\sigma}$ we find that $(\Delta \sigma)=\hat{\sigma}$. The seismic moment (19) of a simple fault is larger than that of a complex fault (18) by the ratio of the total radius $R$ to that of a typical subevent $\rho$. Similar results would be obtained for faults where isolated patches of the fault remain unbroken after the event. Simple smooth faults have larger seismic moments than complex ones with similar stress drops on the broken patches. This is natural, since the unbroken patches appear to 'pin down' the fault, reducing the total fault slip.

The problem of interest in seismology is usually the inverse one: what is the stress drop in the broken patches of the fault? For given $M_{0}$ and $S$ and using the usual circular fault model, the stress drop would be estimated as

$$
\langle\Delta \sigma\rangle=\hat{\sigma} \rho / R
$$

This is the stress drop that would be estimated if the usual assumption of smooth faulting is made. It is clear that $\langle\Delta \sigma\rangle$ underestimates the true stress drop on the broken patches of the fault by a factor $\rho / R$, the ratio of the typical radius of a subfault to the total fault radius.

\section{Three Numerical Models}

In the following we shall study numerical solutions to three models of rupture that illustrate the foregoing discussion. Let us consider a rectangular fault as shown in Figure 2. As in Haskell's model we shall assume that rupture starts suddenly at $x=0$ over the whole width $W$ of the fault. It extends subsequently at a constant rupture velocity $v_{R}=0.75 v_{S}$, where $v_{S}$ is the shear wave velocity. The length of the fault, $L=2 W$, is the same in all three cases. Model $I$ is a simple smooth fault on which the stress drop $\Delta \sigma$ is uniform (Figure $3 a$ ). This model is essentially Haskell's but with constant stress drop rather than constant source time function on the fault. Model II is also a simple fault but with nonuniform stress drop (Figure $3 b$ ). The stress drop varies only as a function of $x$, i.e., it is uniform on any given cross section of the fault. The fault plane is divided into three sections of equal length $L / 3$. In the central section we assume that the stress has already been relieved and the stress drop is zero, but slip may occur freely. In the two extreme sections the stress drop is $1.7 \Delta \sigma$. This value of the stress drop is used in order to get the same seismic moment for all three models. The extreme sections may be considered as asperities where a large stress is released during the earthquake. Model III (Figure 3c) is a complex event where the central one tenth of the fault does not break. For simplicity we assume that in the unbroken section a virtual rupture continues propagating at velocity $v_{R}$, so that the new rupture is nucleated at $x=0.55 L$ at time $\mathrm{t}=0.55 L / v_{R}$. This model corresponds closely to model PSV1 of Das and Aki [1977]. The main differences are that our calculation is three-dimensional, while theirs is two-dimensional and that we fixed the rupture velocity, while they calculated it from a rupture criterion. This cannot be done in our calculations, since the fault would tend to grow faster near the center, thereby loosing its simple rectangular shape. Renormalizing the static solution, so that the seismic moment is the same as in model $I$, we find that the required stress drop in the broken segments is $2.0 \Delta \sigma$. This result may be interpreted with the theory of the last sections. In order to generate the same seismic moment, a complex fault with unbroken segments needs a larger stress drop in its broken parts. Model II is intermediate between models I and III; its average stress drop is $1.12 \Delta \sigma$, only slightly larger than the uniform stress drop of the first model. This small difference is due to the border effects of the faults, which cause stress drops

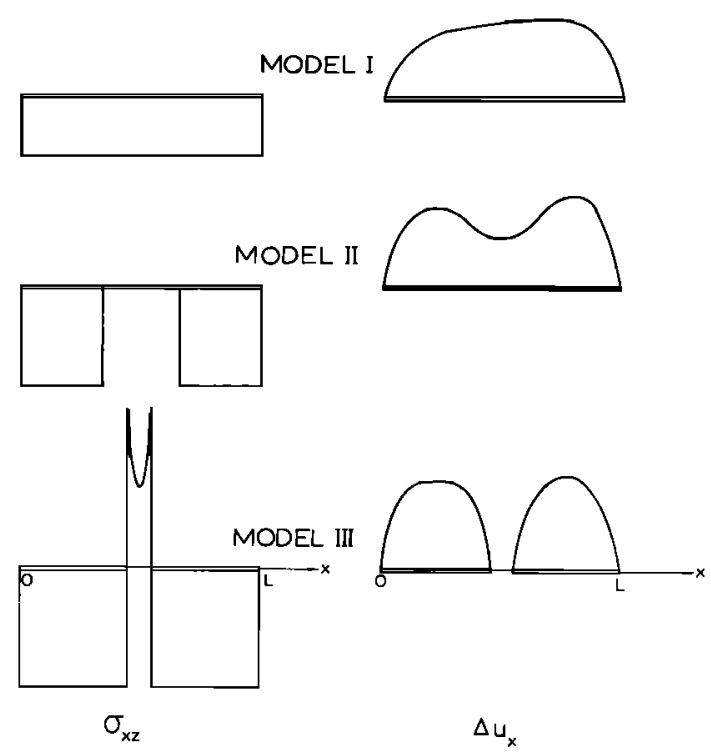

Fig. 3. The three models of rupture studied in the text. The figures show (left) the stress drop, and (right) the final slip along the axis $y=0$ of the fault. Stresses and slips are normalized so that the three models have the same seismic moment. (a) In model I the stress drop is uniform. (b) In model II the stress drop is zero in the central one third of the fault. Slip occurs everywhere on the fault plane but it is reduced in the section with no stress drop. (c) Model III is a double model, where the central one tenth of the fault does not break. The unbroken section develops a large stress concentration (stress increases). This stress was obtained from the final static configuration of the fault system. 

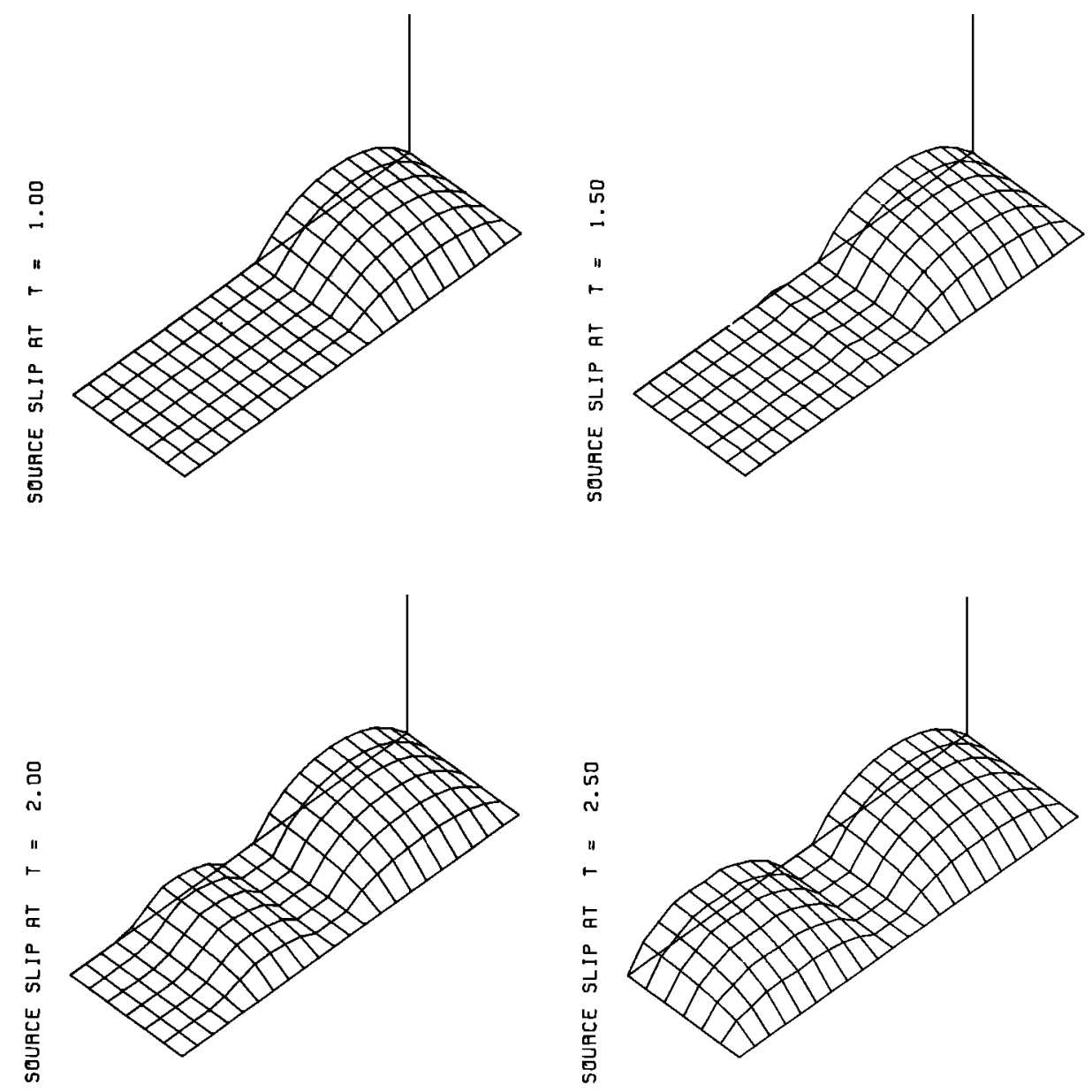

Fig. 4. Slip on the fault for model III shown at four instants of time. At $t=1$, rupture is advancing in the first fault. At $t$ $=1.5$, rupture of the first block is complete. At $t=2.0$, rupture is progressing in the second fault. At $t=2.5$, rupture has already been concluded, and the final static slip has already been reached. Time is measured in units of $2 L / V_{p}$.

near the edges to have less weight in calculating the seismic moment (equation (13)).

It appears then that these three models with widely different stress distributions have the same seismic moment. If we wanted to distinguish between them, we would have to look at higher frequencies or to details of the radiation. This requires dynamic solutions of those models. We have done that using the three-dimensional finite difference method described by $\mathbf{R}$. Madariaga (manuscript in preparation, 1978). This program calculates the history of slip on the fault. As an example, in Figure 4 we show the slip on the fault at four different instants of time for model III. In the first instant we show the slip before rupture arrives at the barrier. In the second, the first subfault is entirely broken. In the third, rupture is already progressing in the second fault. The fourth figure shows the slip after the fault has reached its final static distribution. From this solution and similar ones for the other models we may compute far-field radiation. This is done by means of the representation theorem. Figure 5 shows the far-field pulses and spectra for the three models. For this example we have chosen a direction of radiation with cosine directors $\nu_{x}=\nu_{y}=0.5$ and $\nu_{z}=0.71$. This is typical of radiation in the forward direction with respect to rupture growth. For radiation in the backward direction (i.e., $\nu_{x}<0$ ) the pulses get wider. Let us remark first that $P$ pulses are systematically longer than $S$ pulses. This is typical of unilateral ruptures and is the reverse of what is seen in symmetrical faults, for example, in circular cracks [Mada- riaga. 1977]. Otherwise, $P$ and $S$ pulses are very similar, and we shall refer in the following only to $S$ pulses. The total time length of the pulses in all three cases is close to $T=1.5 \mathrm{~L} / \mathrm{v}_{\mathrm{g}}$ for $S$ waves, indicating that $T$ is related to the overall faulting process. For models II and III the far-field signals show double pulses. The duration of each of these pulses is close to $0.4 L / v_{S}$ and is related to the rupture time through the asperities or subfaults. The radiation from models II and III is quite similar except for the deeper minimum in the pulses of model III. The spectra show characteristics which derive directly from the pulse shapes. For model I, the spectra have simple $f^{-2}$ highfrequency trends and a corner frequency $f_{0} s=0.45 v_{s} / L$. The coefficient 0.45 may seem larger than those in the usual cornerfrequency to size relationships. But notice that here $L$ is the total length, not the radius as in the usual formulas. The spectra for models II and III are more complicated. The main feature is a large secondary spectral lobe. The spectra have a poorly defined intermediate slope and then a steep $f^{-a}$ trend at high frequencies. The estimation of corner frequencies poses some problems; if we take the intersection of the $f^{-3}$ trend with the flat, low-frequency trend, we get a corner frequency $f_{0}^{\prime}=$ $1.2 v_{\mathrm{s}} / L$. This corner frequency is about 3 times that of model I. It is clearly related to the length of the subfaults or asperities. There is no definite corner frequency associated with the overall length of the fault unless we want to introduce an intermediate-frequency trend between the first two spectral lobes. Models II and III are very similar from the point of view 

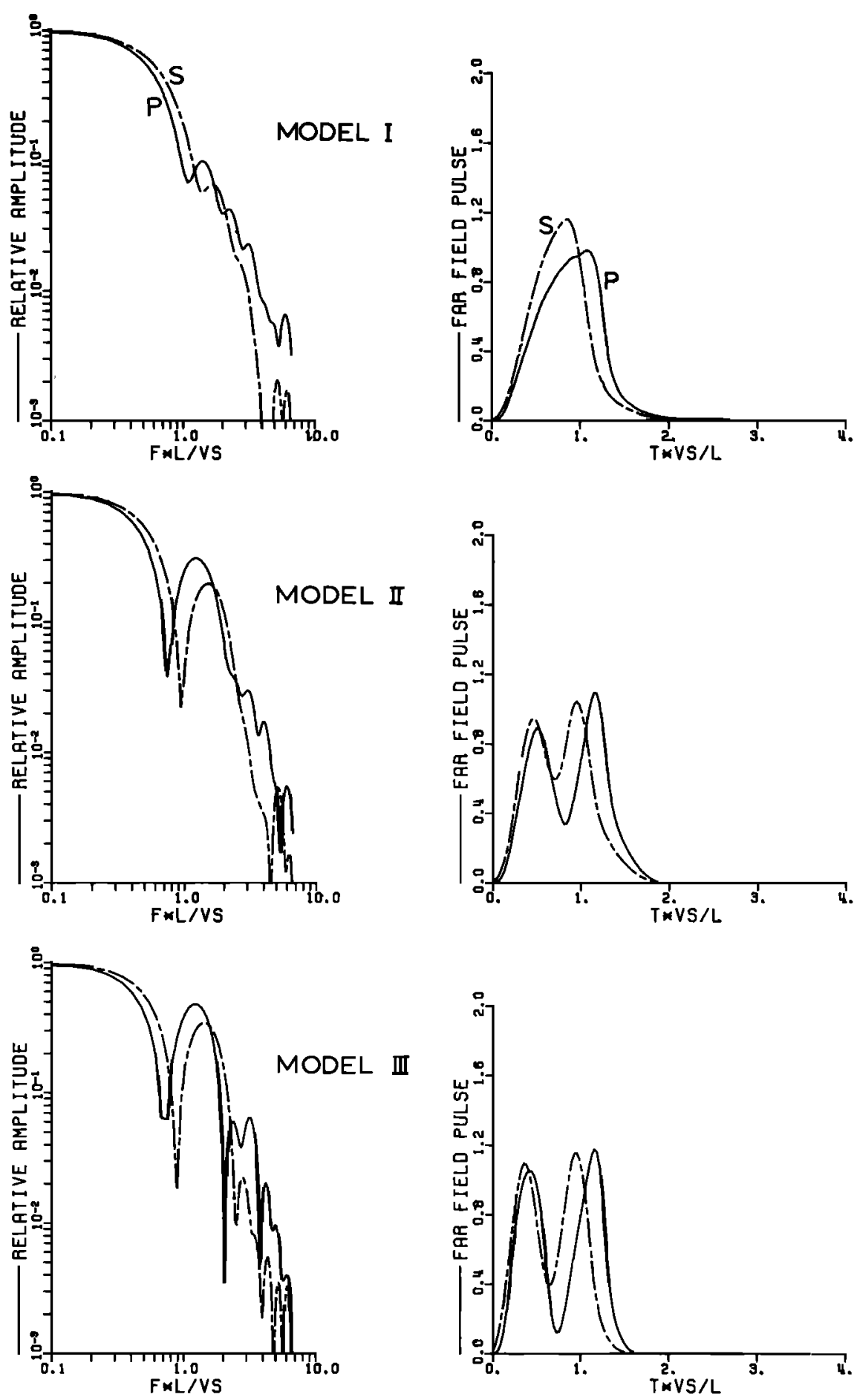

Fig. 5. Radiation from the three models shown in Figure 2. $P$ waves are shown with full traces, $S$ waves with dashed lines. Radiation is shown here for a direction with cosine directors $\nu_{x}=\nu_{y}=0.5$ and $\nu_{z}=0.71$.

of far-field spectra. The numerical solutions presented here are valid at intermediate frequencies; it is probably necessary to look at even higher frequencies to detect significant spectral differences between the two models.

\section{Complex Events Considered as Simple Ones}

Model II and Model III generate very similar far-field pulses, the main difference being the deeper minimum in the model III pulses. In this respect, model II appears as a less extreme example of source complexity than model III is. In fact it is possible to interprete model III as a simple rectangu- lar fault with a highly heterogeneous stress drop distribution. In the central, unbroken section the stress drop is negative or, rather, there is a stress rise in this section. This stress rise is exactly the stress concentration necessary to cancel slip in this segment. This stress is, of course, unknown a priori; it can only be computed as part of the solution to the complex crack problem. In Figure $3 c$ we show the stress concentration calculated on the $y=0$ line, its most significant feature being the inverse square root stress concentrations typical of crack problems. This stress concentration is a function of $y$; in particular, as $y \rightarrow \pm W$, the square root singularities disappear. We may 
calculate now the estimated stress drop $\langle\Delta \sigma\rangle$ assuming that the fault is a simple one using (13). Due to the large negative stress drops in the unbroken section, $(\Delta \sigma)$ is significantly lower than the stress drop in the broken segments. This model allows us to consider complex events as simple ones but is not very effective computationally, since it requires solving the complete crack problem in order to find the stress concentration in the unbroken segments. The relations we have derived in the previous sections evaluate the seismic moment from a knowledge of the stress drop in the broken segments only. We can understand now why model II and model III are similar. In model III the stress drop is large and negative in the central section, while in model II it is exactly zero there. This explains why model III has a deeper minimum in the far-field pulses of Figure 5.

This model is also useful in more general situations where flat, isolated fault segments are not the only features of the earthquake. Since large earthquake faults are probably very complicated, with kinks, barriers, material property discontinuities, etc., it is quite likely that certain segments of the fault do not fail completely during the main event. There might be sections between the component faults which deform plastically. In most cases these defects or barriers of the fault will block or reduce slip and thereby generate stress concentrations. The double fault of model III with a perfectly elastic barrier between them is an idealization. In practice the barrier may suffer plastic deformation. This would reduce the stress concentrations somewhat and increase the estimated stress drop $\langle\Delta \sigma\rangle$; but the main conclusion is still that source heterogeneity in the form of barriers, preslip segments, etc. will tend to yield an estimated stress drop that is a lower bound to the stress drop in the segments that actually broke during the earthquake.

\section{Source Volume and Source Strain}

An examination of the definitions (12), (18), or (19) reveals that the seismic moment is actually the product of the estimated stress drop by a certain volume. Thus, although the fault is plane, a volume appears naturally in the definition of the seismic moment in terms of the stress drop. This volume may be written in the general form

$$
V=C_{l} l S
$$

where $C_{l}$ is a constant, $S$ is the source area, and $l$ is a characteristic length. For simple smooth faults, $l$ is the half-width $W$ of the fault (equation (12)). For a circular fault $l=R$, the radius of the fault, and $V=2.28 R^{3}$. For complex faults, (18) indicates that $l=\rho$, the typical radius of a subfault. We may in general define $l$ as the typical length of the freely slipping segments of the fault. This length appears naturally in (21), since the slip on a crack is always controlled by the shortest dimension on the fault. Physically, we may interpret this volume as the size of the region where large deformation occurs (of order $\Delta \sigma / \mu$ ). In this sense, (21) may be used as a definition of the source volume associated with a plane fault. For complex events this volume is reduced by a factor of $l / R$ compared to that of a simple smooth fault.

It is interesting to note at this point that the estimated stress drop $\langle\Delta \sigma\rangle$ is closely related to the definition of plastic strain used in dislocation theory. In fact, the source strain $\epsilon^{P}=\langle\Delta \sigma\rangle /$ $\mu$ corresponds to the plastic strain as defined by $M c$ Clintock and Argon [1966, p. 105]. The seismic moment may then be defined as

$$
M_{0}=\mu \epsilon^{P} V=\langle\Delta \sigma\rangle V
$$

This relation in fact generalizes the usual definition (1) of the seismic moment, since here it is not necessarily related to slip on a simple plane fault. It may, for instance, be due to slip on a series of faults distributed in a volume as discussed by Kostrov [1974]. It may also be related to general inelastic deformation in the source region as discussed by Randall [1971], Backus and Mulcahy [1976], and McGarr [1976].

\section{CONCLUSIONS}

The seismic moment was introduced as a kinematic parameter describing the radiation of low-frequency seismic waves by a dislocation source. From the representation theorem it was found that it is a measure of the average slip discontinuity on the fault multiplied by the source area. In this sense it is a measure of the distortion of the source region due to faulting. We have shown in this paper that it may also be given a dynamic interpretation in terms of the stress drop of the fractured areas at the source. The relation is very general but not as simple as that between moment and slip at the source. In fact, the geometry of the source affects the seismic moment as much as the stress drop itself. In particular, for multiple sources the seismic moment is smaller than for simple faults of the same area and stress drop. A simple interpretation of this result is that the unbroken sections of the fault pin down the fault and reduce the distortion of the source region, reducing in consequence the seismic moment which measures this distortion. Consequences for the inverse problem are severe: the stress drops for complex events, estimated by the usual circular fault formula [Keilis-Borok, 1959], are only a fraction of the actual stress drop on the broken sections of the faults. The actual and estimated stress drops are in the ratio of the total source size to the size of a typical subevent.

Observations of many earthquakes have led to the conclusion that the stress drops are almost constant ranging from about 10 to 100 bars [Aki, 1972; Kanamori and Anderson, 1975; Hanks, 1977]. These stress drops have been obtained from formulas derived from simple smooth faults. There is abundant evidence that many events are not simple but rather complex, multiple events. In this case our previous discussion implies that the actual stress drops, at least in some segments of the fault, are probably much larger than estimated by the usual methods. This has already been proposed and documented by several authors, including Hanks [1974], Burdick and Mellman [1976], and Aki et al. [1979]. We conclude that on the large scale the stress drop is nearly constant from event to event; but on a smaller scale the stress drop may display large variations on the fault plane. In this small scale the stress drop may not only vary but it may be very large or even negative (stress increase) as in the case of model III, the barrier model of Das and Aki [1977].

Acknowledgments. Discussions and preprints from K. Aki were very helpful. A. Dahlen, J. P. Poirier, and R. Dmowska provided very useful discussion. T. Hanks made extremely valuable suggestions on the original draft. This work was done at the L.E.G.S.P. associated with Centre National de Recherche Scientifique grant 195 and was partially supported by INAG under ATP grant 30-35. Publication 310 of the Institut de Physique du Globe.

\section{REFERENCES}

Aki, K., Generation and propagation of $G$ waves from the Niigata earthquake of June 16, 1964, 2, Estimation of earthquake moment, released energy, and stress-strain drop from $G$-waves spectrum, Bull. Earthquake Res. Inst. Tokyo Univ., 44, 73-88, 1966.

Aki, K., Earthquake mechanism, Tectonophysics, 13, 423-446, 1972. Aki, K., M. Bouchon, B. Chouet and S. Das, Quantitative prediction 
of strong motion for a potential earthquake fault, Ann. Geofis., in press, 1979.

Backus, G. and M. Mulcahy, Moment tensors and other phenomenological descriptions of seismic sources, I, Continuous displacements, Geophys. J. Roy. Astron. Soc., 46, 341-362, 1976.

Burdick, L. J., and G. R. Mellman, Inversion of the body waves of the Borrego Mountain earthquake to the source mechanism, Bull Seismol. Soc. Amer., 66, 1485-1499, 1976.

Burridge, R., and L. Knopoff, Body force equivalents for seismic dislocations, Bull. Seismol. Soc. Amer., 54, 1875-1888, 1964.

Das, S., and K. Aki, Fault plane with barriers: A versatile earthquake model, J. Geophys. Res., 82, 5658-5670, 1977.

Eshelby, J. D., The determination of the elastic field of an ellipsoidal inclusion, and related problems, Proc. Roy. Soc., Ser. A, 241, 376$391,1957$.

Fukao, Y., and M. Furumoto, Foreshocks and multiple shocks of large earthquakes, Phys. Earth Planet. Interiors, 7, 143-153, 1975.

Hanks, T. T., The faulting mechanism of the San Fernando earthquake, J. Geophys. Res., 79, 1215-1229, 1974.

Hanks, T. C., Earthquake stress drops, ambient tectonic stresses, and stresses that drive plate motions, Pure Appl. Geophys., 115, 441-458, 1977.

Kanamori, H., The energy release in great earthquakes, $J$. Geophys. Res., 82, 2981-2987, 1977.

Kanamori, H., and D. L. Anderson, Theoretical basis of some empirical relations in seismology, Bull. Seismol. Soc. Amer., 65, 10731095,1975

Kanamori, H., and G. S. Stewart, Seismological aspects of the Guatemala earthquate of February 4, 1976, J. Geophys. Res., 83, 34273434, 1978.

Keilis-Borok, V. I., On the estimation of the displacement in an earthquake source and of source dimensions, Ann. Geofis., 12, 205$214,1959$.
Knopoff', L., Energy release in earthquakes, Geophys. J. Roy. Astron. Soc., l, 44-52, 1958.

Kostrov, B. V., Seismic moment and energy of earthquakes, and seismic flow of rock, Izv. Acad. Sci. USSR Phys. Solid Earth, 23-44, 1974.

Madariaga, R., Implications of stress-drop models of earthquakes for the inversion of stress drop from seismic observations, Pure Appl. Geophys., 115. 301-316, 1977.

McClintock, F. A., and A. S. Argon, Mechanical Behaviour of Materials, Addison-Wesley, Reading, Mass., 1966.

McGarr, A., Seismic moments and volume changes, J. Geophys. Res., $81,1487-1494,1976$.

Mikumo, T., and T. Miyatake, Dynamical rupture process on a threedimensional fault with non uniform friction and near-field seismic waves, Geophys. J. Roy. Astron. Soc., 54, 417-438, 1978.

Randall, M. J., Shear invariant and seismic moment for deep focus earthquakes, J. Geophys. Res., 76, 4991-4992, 1971.

Savage, J. C., Steketee's paradox, Bull. Seismol. Soc. Amer., 59, 381384, 1969.

Spottiswoode, S. M., and A. McGarr, Source parameters of tremors in a deep-level gold mine, Bull. Seismol. Soc. Amer., 65, 93-112, 1975.

Thatcher, W., and T. Hanks, Source parameters of southern California earthquakes, J. Geophys. Res., 78, 8547-8576, 1973.

Wu, F. T., and H. Kanamori, Source mechanism of February 4, 1965, Rat Island earthquake, J. Geophys. Res., 78, 6082-6092, 1975.

Wyss, M., and J. B. Brune, The Alaska earthquake of March 28, 1964, A complex multiple event, Bull. Seismol. Soc. Amer., 57, 1017-1023, 1967.

(Received May 4, 1978;

revised August 29, 1978;

accepted September 7, 1978.) 